\title{
The influence of locomotion on air-sac pressures in little penguins
}

\author{
D. F. Boggs ${ }^{1}, *$, R. V. Baudinette ${ }^{2}$, P. B. Frappell ${ }^{3}$ and P. J. Butler ${ }^{4}$ \\ ${ }^{1}$ Department of Biology, Hall of Sciences 258, Eastern Washington University, Cheney, WA 99004, USA, \\ ${ }^{2}$ Department of Environmental Biology, University of Adelaide, Adelaide, South Australia 5005, Australia, \\ ${ }^{3}$ Department of Zoology, La Trobe University, Melbourne, Victoria 3083, Australia and ${ }^{4}$ School of Biosciences, \\ The University of Birmingham, Birmingham B15 2TT, UK \\ *e-mail: dboggs@ewu.edu
}

Accepted 27 July 2001

\begin{abstract}
Summary
Air-sac pressures have been reported to oscillate with wing beat in flying magpies and with foot paddling in diving ducks. We sought to determine the impact on airsac pressure of wing beats during swimming and of the step cycle during walking in little penguins (Eudyptula minor). Fluctuations averaged $0.16 \pm 0.06 \mathrm{kPa}$ in the interclavicular air sacs, but only $0.06 \pm 0.04 \mathrm{kPa}$ in the posterior thoracic sac, generating a small differential

supracoracoideus muscles. There was no abdominal muscle activity associated with swimming or exhalation, but the abdominal muscles were active with the step cycle in walking penguins, and oscillations in posterior air-sac pressure $(0.08 \pm 0.038 \mathrm{kPa})$ occurred with steps. We conclude that high-frequency oscillations in differential air-sac pressure enhance access to and utilization of the $\mathrm{O}_{2}$ stores in the air sacs during a dive.
\end{abstract} pressure between sacs of $0.06 \pm 0.02 \mathrm{kPa}$ (means \pm S.E.M., $N=4)$. These fluctuations occurred at approximately $3 \mathrm{~Hz}$ and corresponded to wing beats during swimming, indicated by electromyograms from the pectoralis and
Key words: little penguin, Eudyptula minor, locomotion, air-sac pressure, diving, walking.

\section{Introduction}

Changes in air-sac pressures associated with the wingbeat cycle have been demonstrated in flying magpies (Pica pica) (Boggs et al., 1997a), such that the downstroke has a compressive effect and the upstroke an expansive effect, with corresponding increases and decreases in pressure in the air sacs relative to atmospheric pressure. These mechanical effects of locomotion on respiratory system function stimulate a coordination between respiratory and wingbeat cycles during flight to minimize interference and maximize assistance by the wingbeat cycle to the inspiratory and expiratory phases of the breathing cycle (Boggs et al., 1997b). Terrestrial locomotion, i.e. running or waddling, in birds can also alter air-sac pressures (Boggs et al., 1999; Boggs et al., 1998; Brackenbury and Avery, 1980). In all these situations, the respiratory system is open to the atmosphere through an open glottis. However, these investigations raised the possibility, suggested by Eliassen (Eliassen, 1960), that locomotion in diving birds might have sufficient impact on air-sac pressures in the situation when the glottis is closed during diving to enhance mixing of air-sac air with lung air.

This was confirmed in diving tufted ducks (Aythya fuligula), which propel themselves by synchronous foot paddling (Boggs et al., 1998). Differential pressures between the posterior and anterior sacs fluctuated markedly with the paddle stroke. This pressure oscillation appeared to be due, at least in part, to contractions of the abdominal muscles associated with the power stroke that increased pressures in the posterior sac more than in the anterior sacs, thus creating a differential pressure fluctuation with an average magnitude of $0.49 \mathrm{kPa}$ at a frequency of approximately $2.5 \mathrm{~Hz}$. With $50 \%$ of the oxygen stores supporting aerobic dives residing in the respiratory system (Keijer and Butler, 1982), this impact of locomotion on the differential air-sac pressures seems likely to enhance the movement of oxygen from the air sacs into the lungs.

Penguins are even better adapted to an aquatic life and diving behavior than the diving ducks, both morphologically and physiologically, and propel themselves with their wings. Although the respiratory component of their body oxygen store may be smaller, e.g. 32-45\% in Adélie penguins (Pygosalis adeliae) (Culik et al., 1994; Kooyman and Ponganis, 1998) or as little as $23 \%$ in deep-diving king penguins (Aptenodytes patagonicus) (Ponganis et al., 1999), it is nonetheless important. It is clear, from air samples taken from the first exhalation of Humboldt penguins (Spheniscus humboldti) upon surfacing, that oxygen from the respiratory system had been used during the preceding dive (Butler and Woakes, 1984).

In the present investigation, we sought to determine whether wing strokes affect air-sac pressures in submerged swimming penguins and whether the abdominal muscles play any role, as they do in diving ducks. To this end, we measured upstroke, 
downstroke and abdominal muscle activity patterns together with anterior and posterior air-sac pressures in little penguins (Eudyptula minor) swimming in a water flume. We also recorded abdominal muscle activity and posterior thoracic airsac pressures from penguins walking on a treadmill.

\section{Materials and methods}

Little penguins (Eudyptula minor Forster) were collected from Kangaroo Island in South Australia and transported to the animal facility at the University of Adelaide, where they were housed with free access to water and artificial burrows and were fed fish daily. Those penguins that proved willing to swim and improved in swimming in the water flume over a week of training were selected as the experimental animals. Data were collected successfully from four individuals (body mass $1.09 \pm 0.08 \mathrm{~kg}$ ) while swimming in the water flume and from five $(1.11 \pm 0.08 \mathrm{~kg})$ (means \pm S.E.M.) while walking on a treadmill.

The water flume was of the recirculating type based on the design of Vogel (Vogel, 1981) as described by Fish and Baudinette (Fish and Baudinette, 1999). The maximum swimming speed achievable in this flume was $0.95 \mathrm{~m} \mathrm{~s}^{-1}$. The treadmill had a motor-driven tread of $1.25 \mathrm{~m} \times 0.35 \mathrm{~m}$, and the penguins walked on it at a speed of $0.4 \mathrm{~m} \mathrm{~s}^{-1}$.

The penguins were instrumented while anesthetized with 2-4\% flurothane in oxygen. Bipolar silver electromyographic (EMG) electrodes $[0.1 \mathrm{~mm}$ insulated $0.99 \%$ silver wire (California Fine Wire Co.) with $1 \mathrm{~mm}$ of the tip bared] were introduced via a 26-gauge needle into the pectoralis (downstroke) muscle, the supracoracoideus (upstroke) muscle and an abdominal muscle (internal oblique). The wires were tunneled under the skin to a microconnector (Microtech GM6) plugged into a cable (Cooner Wire Co., NMUF-6/30-40462J). The signals were passed to preamplifiers (Grass model 7P122). The interclavicular and posterior thoracic air sacs were cannulated with either PE200 or silastic tubing (internal diameter $1.6 \mathrm{~mm}$, external diameter $3.18 \mathrm{~mm}$ ) with side holes, held in place with the aid of superglue. Silastic tubing was tunneled under the skin to exit the bird at the base of the skull. During recordings, these air-sac cannulae were connected to Grass PT5 pressure transducers for separate air-sac pressure recordings and to both sides of a miniature piezoresistive differential pressure transducer (Endevco, 8507C-2) for differential pressure recordings between the anterior and posterior air sacs. The tubing connecting the air-sac catheters to the pressure transducers mounted above the water flume was rigid polyethylene tubing $2 \mathrm{~mm}$ in internal diameter and $1.2 \mathrm{~m}$ long.

The $95 \%$ response time of the PT5 transducers in this arrangement was $0.1 \mathrm{~s}$, and that of the Endevco differential pressure transducer was $0.07 \mathrm{~s}$. These are longer than the manufacturer's specifications for the transducers, and the tubing therefore introduced some delay into the pressure signal response, but not enough to compromise recordings of events that were of significantly longer duration. The potential sources of error in the differential pressure measurements were analyzed in a previous study of diving ducks (Boggs et al., 1998). The intrinsic error in the differential pressure measurements is only of the order of $0.7 \%$. The principal potentially significant source of error in such measurements comes from the presence of fluid in one or other of the two airsac catheters [see (Boggs et al., 1998)]. Knowing this, we were vigilant about watching for fluid in the tubing, frequently 'blew out' the tubing and rejected far more data than we accepted because of fluid accumulation in one of the catheters.

While the penguin swam in the water flume or walked on the treadmill, all the signals from pressure transducers and EMG electrodes were transmitted to a TEAC MR40 instrumentation tape recorder, a Grass chart recorder and a computer with the Grass Polyview data-acquisition system. The EMG recordings from the pectoralis and supracoracoideus muscles were used to identify the phases of the wingbeat cycle; for the treadmill walking sequences, an observer pushed an event marker with each footfall of the left foot of the penguin.

After data collection, the penguins were re-anesthetized, all tubes and wires were removed and the birds were allowed to recover for 2 weeks before being returned to the colony on Kangaroo Island.

Because we were not permitted to implant arterial catheters and collect blood samples from the penguins, we later used four mature Muscovy ducks (Cairina moschata, L.) (mass $3.02 \pm 0.37 \mathrm{~kg}$ ) to assess the effects on arterial blood gas levels of oscillations in differential pressure of similar magnitude to those we observed in the penguins. The ducks were anesthetized with $25 \mathrm{mg} \mathrm{kg}^{-1}$ Ketamine and $2 \mathrm{mg} \mathrm{kg}^{-1}$ Xylaxine intramuscularly. The left brachial artery and vein were cannulated with PE90 tubing. An endotracheal tube was inserted into the trachea and attached to a Harvard Apparatus ventilator and, via a T-tube, to a pressure transducer (Endevco $8075 \mathrm{C})$. The duck was paralyzed with gallamine triethiodide ( $1 \mathrm{mg} \mathrm{kg}^{-1}$, intravenously) and ventilated to achieve good oxygenation and moderate hypocapnia. Arterial blood samples $(250 \mu \mathrm{l})$ were withdrawn while the duck was ventilated and again after $45 \mathrm{~s}$ of no ventilation with the endotracheal tube clamped. This period falls within the range of dive times observed in free-swimming little penguins and tufted ducks (Aythya fuligula) (20-46s) (Gales et al., 1990; Stephenson et al., 1986; Butler and Jones, 1997). This protocol was followed with no chest wall oscillation and again with manual sternal oscillation at $3 \mathrm{~Hz}$, to achieve either small pressures close to the average differential pressure fluctuation recorded from submerged swimming penguins $(0.07 \mathrm{kPa})$ or higher pressures approaching those reported for diving ducks $(0.3 \mathrm{kPa})$. Each of these three protocols was performed at least twice in each of the four ducks. Blood gas levels were measured with a Radiometer BMS MK2 blood gas analyzer at $37^{\circ} \mathrm{C}$ and corrected to the duck's body temperature $\left(39-40^{\circ} \mathrm{C}\right)$ measured by a colonic YSI thermistor (using the correction factors of Severinghaus, 1966).

We applied paired $t$-tests to determine whether there was any significant difference in the mean time intervals between air- 
Table 1. Comparative effects of locomotion on air-sac pressures

\begin{tabular}{|c|c|c|c|c|c|c|}
\hline & \multirow[b]{2}{*}{$N$} & \multicolumn{2}{|c|}{$\begin{array}{c}\Delta \text { Absolute pressure } \\
(\mathrm{kPa} \text {; relative to atmospheric) }\end{array}$} & \multirow{2}{*}{$\begin{array}{c}\Delta \text { Differential pressure } \\
\text { (KpA; i.e. between sacs) }\end{array}$} & \multicolumn{2}{|c|}{$\begin{array}{l}\text { Driving pressure per breath } \\
(\mathrm{kPa})\end{array}$} \\
\hline & & Anterior & Posterior & & Anterior & Posterior \\
\hline $\begin{array}{l}\text { Swimming } \\
\text { Penguin } \\
\text { Tufted duck }{ }^{1}\end{array}$ & $\begin{array}{r}24 \\
5\end{array}$ & $0.115 \pm 0.06$ & $0.059 \pm 0.04$ & $\begin{array}{c}0.061 \pm 0.022 \\
0.49 \pm 0.13\end{array}$ & & $\begin{array}{c}0.31 \pm 0.04 \\
0.133\end{array}$ \\
\hline $\begin{array}{l}\text { Flying } \\
\text { Magpie }^{2}\end{array}$ & 5 & & $0.37 \pm 0.13$ & & $0.43 \pm 0.063$ & $0.50 \pm 0.056$ \\
\hline $\begin{array}{l}\text { Walking } \\
\text { Penguin } \\
\text { Tufted duck }{ }^{1} \\
\text { Chukar }^{3}\end{array}$ & $\begin{array}{l}5 \\
5 \\
5\end{array}$ & & $\begin{array}{c}0.08 \pm 0.038 \\
0.105 \\
0.06 \pm 0.017\end{array}$ & & & $\begin{array}{c}0.161 \pm 0.062 \\
0.123 \\
0.115 \pm 0.05\end{array}$ \\
\hline
\end{tabular}

${ }^{1}$ Boggs et al., 1998; ${ }^{2}$ Boggs et al., 1997a; ${ }^{3}$ Boggs et al., 1999.

Values are means \pm S.E.M.

sac pressure oscillations and between either wing beats (from pectoralis EMG activity) in the swimming penguins or steps (from abdominal EMG activity) in the walking penguins. The differences in mean values for arterial $P_{\mathrm{O}_{2}}\left(\mathrm{PaO}_{2}\right)$ in the three different protocols on ducks were assessed by an analysis of variance (ANOVA).

\section{Results}

We observed fluctuations in differential pressure with wing stroke during the brief breath-hold periods (mean 5.3s) of submerged swimming in all four penguins (Fig. 1; Table 1) (mean wingbeat frequency $2.97 \pm 0.2 \mathrm{~Hz}$ ). There was no detectable activity in the abdominal muscles of swimming penguins, but such activity was apparent in the walking penguins (Fig. 1, Fig. 2). The pressures tended to fluctuate more in the anterior (interclavicular) than in the posterior sacs in all the swimming penguins, with a short lag $(0.028 \mathrm{~s})$ in changes in posterior air-sac pressure behind anterior air-sac pressure detectable in two of the penguins. There was no significant difference between the time intervals between successive wingstrokes, as represented by pectoralis EMG activity, and successive air-sac pressure oscillations, supporting the contention that the two are related. Abdominal muscle activity in the

Fig. 1. Differential pressure fluctuations occurring with wing beats indicated by contractions of the pectoralis and supracoracoideus muscles. A breath (marked) is associated with the penguin raising its head above the surface while still swimming. No abdominal muscle activity was apparent during swimming or with the expiratory phase of the breath shown here. walking penguin was not synchronous with expiration but rather with the step cycle.

The temporal inaccuracy of the 'observer' mechanism makes phasic relationships between step cycle and abdominal muscle activity difficult to assess with certainty but does allow the conclusion to be drawn that abdominal muscle activity is associated with the step cycle and not with the expiratory phase of the breath cycle, as it often is in other birds, at rest or during inactive hyperventilation states. There were twice as many fluctuations in pressure in the posterior thoracic air sac (Fig. 2) as there are bursts of EMG activity in the left abdominal muscles, suggesting that the right abdominal muscles were active in phase with the step cycle of the right leg, which was temporally opposite to that on the left leg, and that each step was altering air-sac pressure. There was no significant difference
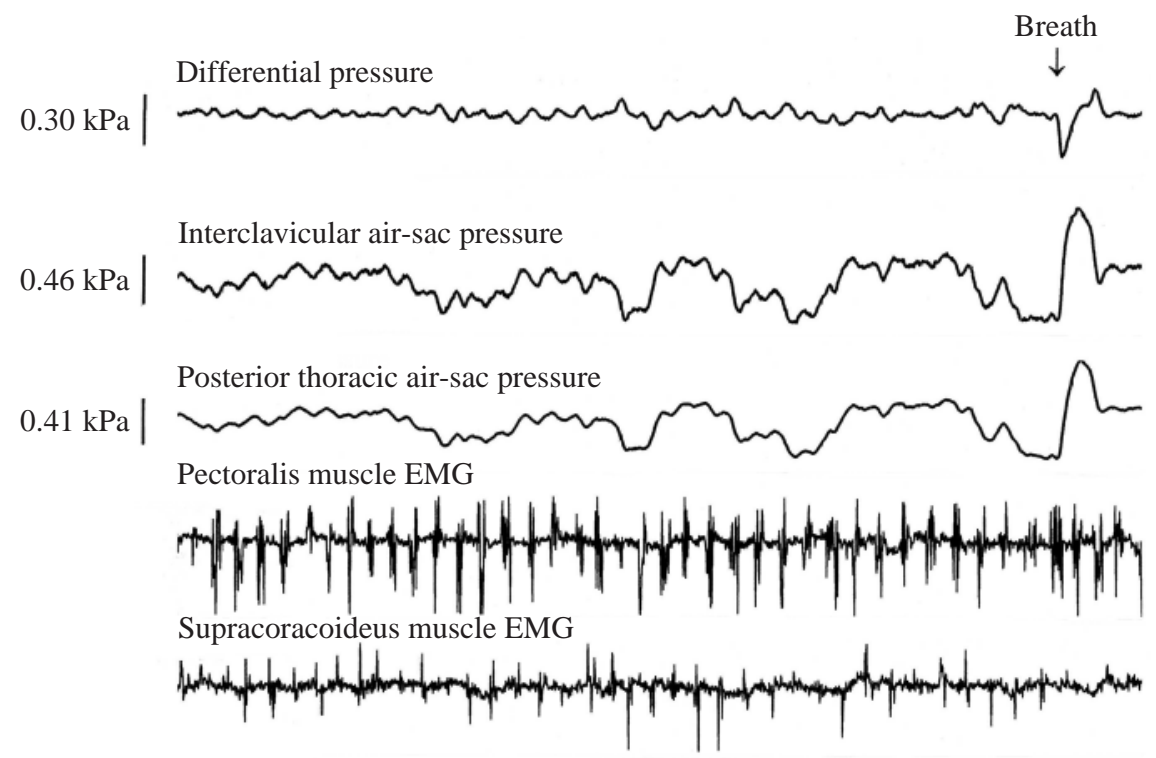

Abdominal muscle EMG

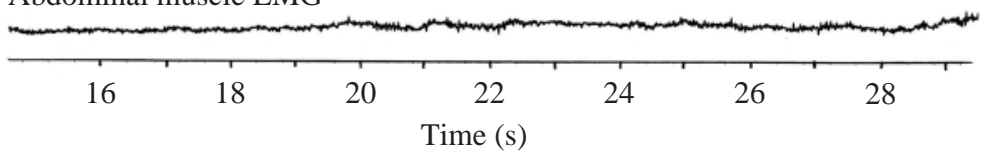


Table 2. Mean decline in arterial $\mathrm{P}_{2}$ after a $45 \mathrm{~s}$ breath-hold with and without chest wall oscillation in Muscovy ducks Cairina moschata

\begin{tabular}{|c|c|c|c|}
\hline & No oscillation & $\begin{array}{c}\text { Oscillation at } \\
3 \mathrm{~Hz} \text {, to } 0.07 \mathrm{kPa}\end{array}$ & $\begin{array}{c}\text { Oscillation at } \\
3 \mathrm{~Hz} \text {, to } 0.3 \mathrm{kPa}\end{array}$ \\
\hline Mean drop in $\mathrm{PaO}_{2}(\mathrm{kPa})$ & $4.4 \pm 0.4$ & $2.77 \pm 0.35$ & $2.37 \pm 0.55$ \\
\hline ANOVA & $\begin{array}{l}P<0.02 \text {; significantly different from } \\
\quad \text { both oscillation protocols }\end{array}$ & $\begin{array}{l}\text { Not significantly different from } \\
\text { other oscillation protocols }\end{array}$ & \\
\hline
\end{tabular}

Fig. 2. Posterior thoracic air-sac pressure exhibits fluctuations with steps as this penguin walks at $0.4 \mathrm{~m} \mathrm{~s}^{-1}$ on a treadmill. Footfall of the left foot was recorded by an observer pushing an event marker (signal down). Abdominal muscle activity is related to step cycle and may contribute to the pressure oscillations.
Posterior thoracic air-sac pressure

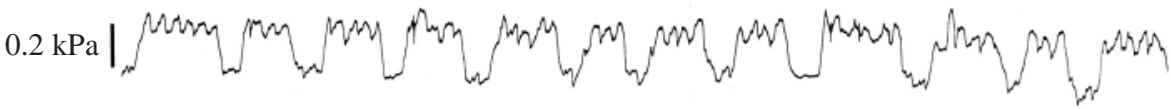

Left abdominal muscle EMG

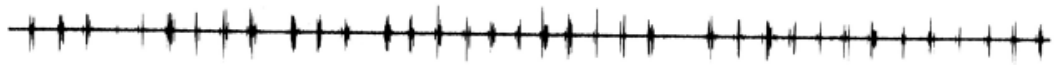

Left footfall $\downarrow$

$1 \mathrm{~s}$ in the time interval between pressure oscillations and $0.5 \times$ the interval between bursts of left abdominal muscle EMG activity, again supporting the concept that there is a relationship between some mechanical events of the stepping cycle and the cycle of air-sac pressure oscillations.

In the Muscovy ducks, arterial $\mathrm{PaO}_{2}$ fell by only approximately 53-61\% as much (mean $2.8 \mathrm{kPa}$ with small pressures, $2.4 \mathrm{kPa}$ with greater pressures) during a breath-hold with sternal oscillation as during a breath-hold without oscillation (4.4 kPa) (Table 2). The magnitude of the decline in $\mathrm{PaO}_{2}$ was not significantly different between the two oscillation protocols, but there was a significantly greater $(P<0.02)$ decline in $\mathrm{PaO}_{2}$ without oscillation compared with either of the oscillation protocols.

\section{Discussion}

\section{Mechanics of air-sac pressure oscillations}

Fluctuations in air-sac pressures and differential pressures associated with wing beats were observed between the anterior and posterior sacs in the swimming penguin. This is qualitatively similar to observations of locomotion-induced changes in air-sac pressures in flying magpies, in swimming and running ducks and in running chukars (Alectoris chukar) and chickens (Boggs et al., 1997a; Boggs et al., 1998; Boggs et al., 1999, Brackenbury and Avery, 1980). However, as indicated in Table 1, the magnitude of the pressure change with wing stroke is greater, by a factor of 3 , in the flying magpie than that in the swimming little penguin. The source of compression and expansion of air sacs in the flying bird was determined radiographically to be movements of the sternum relative to the vertebral column, but the cause of that was not entirely clear (Boggs et al., 1997a). The possibilities seemed to include (i) pectoralis muscle contraction causing not only wing downstroke but movement of the sternum towards the vertebral column, as it moves during expiration, and relaxation allowing it to move away again during inspiration and/or (ii) the lift forces generated by the downstroke providing an upward pressure against the sternum, compressing the air sacs, and then a falling away of the sternum from the vertebral column during the upstroke, expanding the sacs (Boggs et al., 1997a).

The abdominal muscles were silent during swimming in the penguin and, hence, were not contributing to fluctuations in airsac pressures, as they seemed to in the foot-propelled diving duck (Boggs et al., 1998). During walking, however, the abdominal muscles seemed to play a postural role and contract in synchrony with the step cycle on each side. This corresponds to the pressure changes in the air sacs with stepping, as it did in running ducks (Boggs et al., 1999), and pressure changes with steps have also been observed in running chickens (Brackenbury and Avery, 1980). However, in a bird adapted for running, the chukar, the abdominal muscles generally act as expiratory muscles, contracting not in synchrony with stepping but with breathing, and yet there are still oscillations in air-sac pressure with stepping (Boggs et al., 1999). This observation calls into question the relative significance of abdominal muscle activity in generating the pressure 
oscillations with stepping and suggests that the inertial effects of the abdominal contents may be quantitatively more important. It is also interesting to note that the abdominal muscles seem to play little role in ventilation in the little penguin, so ventilation must be driven only by the activity of the intercostal muscles. The abdominal muscles did not become active during the expiratory phase of the breath shown in Fig. 1 or during submerged exhalations. They are often silent during resting breathing in other birds, but usually become active with the hyperventilation of stimulated breathing or with exercise, as in the running chukar or flying magpie (Boggs, 1997; Boggs et al., 1999).

Although the pectoralis is acting as the downstroke muscle in the penguin, as it is in the flying magpie, there are differences in the force vectors, in the amplitude of the wing beat and in the sternal morphology of the swimming penguin compared with a flying bird such as the magpie. There is less lift generation and more forward thrust with downstroke in the penguin compared with flying birds, and the upstroke generates thrust as well (Clark and Bemis, 1979). The lift that is generated is directed upwards and forwards on the downstroke and downwards and forwards on the upstroke. The quantitative differences in air-sac pressure oscillations with wing stroke between flying and swimming birds may reflect a greater contribution of lift forces to air-sac pressure changes in the flying bird versus a primarily muscular contraction effect on sternal position in the swimming bird. Also, although unquantified, wing excursion appears to be much smaller in these penguins than in magpies. This may increase with more forceful contractions of the pectoralis and supracoracoideus muscles when the penguins swim faster, but we could not achieve the usual foraging speeds for this species (1.7-2.4 $\mathrm{m} \mathrm{s}^{-1}$ ) (Gales et al., 1990) in our water flume.

The skeletal morphologies of the penguin and magpie are quite different because of the streamlined body form of the penguin. The sternum is longer and more parallel to the vertebral column in the penguin, with the vertebral and sternal ribs articulating at more acute angles than in the magpie, which has a shorter sternum that forms a more oblique angle with respect to the vertebral column (closer to it anteriorly, further away posteriorly) (Duncker, 1971). The interclavicular air sac is larger relative to the posterior thoracic air sac in the penguin than in the magpie. All these features may contribute to the difference in relative pressure changes observed with the wing stroke in the penguin and magpie: the changes in pressure are greater in the posterior sac than in the anterior sacs in the magpie (and the duck), but the reverse is the case in the penguin.

It is interesting to note in this context that Powell and Hempleman (Powell and Hempleman, 1985) found a higher $P_{\mathrm{CO}_{2}}$ than predicted in the caudal thoracic air sac of two gentoo (Pygoscelis papua) and one chinstrap (Pygoscelis antarctica) penguin. Whereas many birds have neopulmonic parabronchi in which gas exchange can occur during bidirectional flow of air into and out of the caudal sacs, penguins have only paleopulmonic parabronchi and, hence, would be expected to have a low caudal sac $P_{\mathrm{CO}_{2}}$ consistent with unidirectional caudal-to-cranial flow through the lung. Yet the caudal sac $P_{\mathrm{CO}_{2}}$ was $1.5-3.5$ times greater than the value predicted from re-inhaled dead space and cannot be explained by stratification or gas exchange across the air-sac walls. Powell and Hempleman (Powell and Hempleman, 1985) were therefore forced to speculate that airflow may not be caudalto-cranial in all paleopulmonic parabronchi. This is consistent with our recordings of higher pressure changes in the anterior than the posterior sacs, which may create airflow patterns through this respiratory system that do not fit the model for avian lungs based upon measurements in inactive ducks or chickens.

\section{Potential utility of air-sac pressure oscillations}

The fluctuation in differential pressure between the anterior and posterior sacs occurs at a frequency of $3 \mathrm{~Hz}$ in the little penguins in this study. How much air is actually moved by the average $0.061 \mathrm{kPa}$ pressure differential is difficult to predict. However, it is worth noting that during ventilation at rest the driving pressure through the resistance of the airways is small in penguins, as in other birds, and that $0.061 \mathrm{kPa}$ is $50-100 \%$ of the pressure, relative to atmospheric, responsible for moving resting tidal volumes through the open system, but only approximately $24 \%$ of the higher pressure used to drive the higher-velocity airflows of breaths during swimming (Table 1). This simply suggests that this small pressure could induce some airflow through the airways.

What the path of airflow would be in the closed system is also unclear because the mechanisms responsible for the aerodynamic valving that creates unidirectional flow through the avian lung during resting breathing are probably compromised (i.e. flows past the branch point of the ventral mesobronchi off the primary bronchus may become turbulent rather than laminar, and the pressure gradients between the anterior and posterior sacs may be reversed from the standard models describing the aerodynamic valving mechanisms) (Banzett and Lehr, 1982; Butler et al., 1988). However, the direction of flow in a cross-current gas exchanger is not important (Scheid and Piiper, 1972), and the potential effect of enhancing the mixing of air-sac air with lung air can be achieved with flow in either direction through the parabronchi or with unidirectional flow, which would require flow from the anterior sacs to the posterior sacs through the primary bronchus in one phase of the cycle.

The (probably small) air volume movements at high frequencies in the swimming penguins are reminiscent of the high-frequency ventilators that became popular in the late 1980s, especially for ventilating newborn humans. The primary mechanism of the enhanced gas exchange of highfrequency oscillation in pressure is through enhanced diffusion, with relatively small contributions from convective airflows, if the analysis of the fluid mechanics of highfrequency ventilators can be applied to this rather different closed-system situation (Drazen et al., 1984; Watson, 1983). 
Banzett and Lehr (Banzett and Lehr, 1982) reported that highfrequency ventilation (via the open airway) was effective in the chicken, although somewhat less so than in the mammalian system, perhaps because of the differences in structure and airflow dynamics. The most effective frequencies in the chicken were $2-5 \mathrm{~Hz}$. A study of high-frequency chest wall compression in dogs also demonstrated that frequencies in the range of $2-5 \mathrm{~Hz}$ were very effective in sustaining gas exchange, even more so than higher frequencies (Zidulka et al., 1983). The empirical data from our duck chest wall oscillation studies clearly suggest that even small pressure oscillations at $3 \mathrm{~Hz}$ do enhance oxygen uptake from the respiratory system during a breath-hold, even if the mechanism remains incompletely understood in terms of models of the fluid dynamics in the avian respiratory system. This impact of locomotion upon respiratory system function can thus enhance the ability of birds to remain submerged for sufficiently long periods to forage for food without having to rely on anaerobic metabolism.

The depth to which a penguin descends within the water column will clearly determine the degree of compression of all the air sacs. This study focused on submerged swimming near the surface; nonetheless, the larger oscillations in pressure at lower frequency than wing beats notable in Fig. 1 can be attributed to changes in depth within the $1 \mathrm{~m}$ deep water flume. Little penguins do not routinely dive to great depths, but the larger penguin species, king (Aptenodytes patagonicus) and emperor (Aptenodytes forsteri), do. A recent study by Ponganis et al. (Ponganis et al., 1999) suggests that there is less reliance on the respiratory fraction of total body oxygen stores in the deeper-diving species than in the shallower-diving species such as the little penguin. The differential pressure oscillations with wing beat described here would be superimposed on any increase in total pressure and could still enhance diffusion and therefore gas exchange as long as it was occurring in the lung. However, one suggestion for a mechanism protecting deepdiving species from 'the bends' is the prevention of gas exchange at depth by the engorgement of the blood capillaries collapsing the air capillaries in the walls of the parabronchi (Kooyman et al., 1999).

We would like to thank Jayne Skinner for her devoted care of the penguins and assistance in training them to swim in the flume. The assistance of Delbert L. Kilgore Jr in obtaining the blood gas data from ducks was also greatly appreciated. This work was supported by a small faculty research grant from Eastern Washington University, a Distinguished Visiting Scholar Award from the University of Adelaide to D.F.B. and a small grant from the Australian Research Council to R.V.B. and D.F.B. These experiments were conducted with the approval of the Animal Ethics Committee of the University of Adelaide and with appropriate wildlife permits from the Australian Department of Natural Resources.

\section{References}

Banzett, R. B. and Lehr, J. L. (1982). Gas exchange during high-frequency ventilation of the chicken. J. Appl. Physiol. 53, R1418-R1422.

Boggs, D. F. (1997). Coordinated control of respiratory pattern during locomotion in birds. Am. Zool. 37, 41-57.

Boggs, D. F., Butler, P. J., Baudinette, R. V. and Frappell, P. B. (1999). Relationship amongst air-sac pressures, steps and abdominal muscle activity in waddling and running birds. FASEB J. 13, A495.

Boggs, D. F., Butler, P. J. and Wallace, S. (1998). Differential air-sac pressures in diving tufted ducks, Aythya fuligula. J. Exp. Biol. 201, 2665-2668.

Boggs, D. F., Jenkins, F. A. and Dial, K. P. (1997a). The effects of the wingbeat cycle on respiration in the black-billed magpie (Pica pica). J. Exp. Biol. 200, 1403-1412.

Boggs, D. F., Seveyka, J. J., Kilgore, D. L. and Dia, L. K. P. (1997b). Coordination of respiratory cycles with wingbeat cycles in the black-billed magpie (Pica pica). J. Exp. Biol. 200, 1413-1420.

Brackenbury, J. H. and Avery, P. (1980). Energy consumption and ventilatory mechanisms in the exercising fowl. Comp. Biochem. Physiol. 66A, 439-443.

Butler, J. P., Banzett, R. B. and Fredberg, J. J. (1988). Inspiratory valving in avian bronchi: aerodynamic considerations. Respir. Physiol. 72, 241-256.

Butler, P. J. and Jones, D. R. (1997). Physiology of diving of birds and mammals. Physiol. Rev. 77, 837-899.

Butler, P. J. and Woakes, A. J. (1984). Heart rate and aerobic metabolism in Humbolt penguins, Spheniscus humboldti, during voluntary dives. J. Exp. Biol. 108, 419-428.

Clark, B. D. and Bemis, W. (1979). Kinematics of swimming of penguins at the Detroit Zoo. J. Zool., Lond. 188, 411-428.

Culik, B. M., Wilson, R. M. and Bannasch, R. (1994). Underwater swimming at low energetic cost by pygoscelid penguins. J. Exp. Biol. 197, 65-78.

Drazen, J. M., Kramm, R. D. and Slutsky, A. S. (1984). High-frequency ventilation. Physiol. Rev. 64, 515-543.

Duncker, H.-R. (1971). The lung air sac system of birds: A contribution to the functional anatomy of the respiratory apparatus. Adv. Anat. Embryol. Cell Biol. 45, 1-171.

Eliassen, E. (1960). Cardiovascular responses to submersion asphyxia in avian divers. Arbok Univ. I Bergen Mt. (Naturv. Ser.) 2, 1-100.

Fish, F. E. and Baudinette, R. V. (1999). Energetics of locomotion in the Australian water rat (Hydromys chrysogaster): a comparison of swimming and running in a semi-aquatic mammal. J. Exp. Biol. 202, 353-363.

Gales, R., Williams, C. and Ritz, D. (1990). Foraging behavior of the little penguin, Eudyptula minor: initial results and assessment of instrument effect. J. Zool., Lond. 220, 61-85.

Keijer, E. and Butler, P. J. (1982). Volumes of the respiratory and circulatory systems in the tufted and mallard ducks. J. Exp. Biol. 101, 213-220.

Kooyman, G. L. and Ponganis, P. J. (1998). The physiological basis of diving to depth: birds and mammals. Annи. Rev. Physiol. 60, 19-33.

Kooyman, G. L., Ponganis, P. J. and Howard, R. S. (1999). Diving animals. In The Lung at Depth, chapter 14 (ed. C. Lungren and C. Miller), pp. 587-620. New York: Marcel Dekker.

Ponganis, P. J., Kooyman, G. L., Van Dam, R. and LeMaho, Y. (1999). Physiological responses of king penguins during simulated diving to $136 \mathrm{~m}$ depth. J. Exp. Biol. 202, 2819-2822.

Powell, F. L. and Hempleman, S. C. (1985). Source of carbon dioxide in penguin air sacs. Am. J. Physiol. 248, R748-R752.

Scheid, P. and Piiper, J. (1972). Cross-current gas exchange in avian lungs: effects of reversed parabronchial airflow in ducks. Respir. Physiol. 16, 304-312.

Severinghaus, J. W. (1966). Blood gas calculator. J. Appl. Physiol. 21, 1108-1116.

Stephenson, R. L., Butler, P. J. and Woakes, A. J. (1986). Diving behavior and heart rate in tufted ducks (Aythya fuligula). J. Exp. Biol. 126, 341-359.

Vogel, S. (1981). Life in Moving Fluids, p. 298. Princeton, NJ: Princeton University Press.

Watson, E. J. (1983). Diffusion in oscillatory pipe flow. J. Fluid Mech. 133, 233-244.

Zidulka, A. D., Gross, H., Minami, V. and Chang, H. K. (1983). Ventilation by high-frequency chest wall compression in dogs with normal lungs. Am. Rev. Respir. Dis. 127, 709-713. 